\title{
tic\&société
}

Vol. 10, N 2-3 | 2ème semestre 2016 - 1er semestre 2017

Spécial varia

\section{Analyse sociologique et économique du financement participatif. Ressorts et critiques dans le cas du journalisme (2010-2015)}

Guillaume GOASDOUÉ

\section{(2) OpenEdition \\ Journals}

Édition électronique

URL : http://journals.openedition.org/ticetsociete/2154

DOI : 10.4000/ticetsociete.2154

Éditeur

Association ARTIC

Édition imprimée

Pagination : 199-229

Référence électronique

Guillaume GOASDOUÉ, « Analyse sociologique et économique du financement participatif. Ressorts et critiques dans le cas du journalisme (2010-2015) », tic\&société [En ligne], Vol. 10, № 2-3 | 2ème semestre 2016 - 1er semestre 2017, mis en ligne le 30 avril 2017, consulté le 01 mai 2019. URL : http://journals.openedition.org/ticetsociete/2154 ; DOI : 10.4000/ticetsociete.2154 
tic\&société - 10(2-3), 2016-2017

\section{Analyse sociologique et économique du financement participatif. Ressorts et critiques dans le cas du journalisme (2010-2015)}

\section{Guillaume GOASDOUÉ}

Guillaume Goasdoué est docteur en sciences sociales de l'université Paris 2 Panthéon-Assas. Ses champs de recherche concernent l'épistémologie, le journalisme, la réception des actualités, le financement des médias et les effets du numérique (formats informationnels, organisation du travail, modèles économiques).guillaume.goa@gmail.com 
Analyse sociologique et économique du financement participatif. Ressorts et critiques dans le cas du journalisme

(2010-2015)

\section{Analyse sociologique et économique du financement participatif. Ressorts et critiques dans le cas du journalisme (2010-2015)}

Résumé : Le système du financement participatif met en relation des contributeurs individuels et des médias qui cherchent des fonds pour quatre raisons principales: le sauvetage, la diversification, la création, les projets ponctuels. Comment le capital (symbolique, social) accumulé par les porteurs de projet favorise-t-il l'ampleur et l'issue des collectes? De quelles manières la dimension sociale du mécanisme est-elle exploitée par ce type de procédé de collecte de fonds ? D'abord, nous répondrons à ces questions en commençant par discuter des limites de la littérature internationale. Ensuite, nous présenterons nos données pour le secteur de l'information, puis nous traiterons plus spécifiquement des ressorts sociaux du phénomène. Enfin, nous finirons par les aspects liés au travail de recherche de visibilité. Seront ainsi critiquées quelques idées reçues, puis exposées diverses formes d'inégalités entre les médias qui recourent à ce système.

Mots-clés : capital symbolique, capital social, journalisme, financement participatif, économie des médias.

Abstract: Crowdfunding systems connect project initiators and contributors in order to finalize financial arrangements. The media use this system for four main reasons: rescue, diversification, launch, one-time project. The origins of these fundraisers are based largely on the symbolic capital ("capital of recognition") and social capital that the media and journalists can mobilize. First, the international literature and the French context (fifteen cases between 2010 and 2015) will be

discussed. Then, two major aspects will be developed. The first aspect considers the eminently social nature of the mechanism, from the system foundation to its execution through the design of the platform. The second aspect focuses on the issue of (mediatic) visibility. Our analysis will dispel some misconceptions ("crowd", innovative character, "easy money") and remind readers of the unequal nature of the system.

Keywords: symbolic capital, social capital, journalism, crowdfunding, media economy.

Resumen: El sistema de crowdfunding (financiamiento participativo) conecta contribuyentes individuales y medios de 


\section{Guillaume GOASDOUÉ}

comunicación que buscan recaudar fondos por cuatro razones principales: rescate, diversificación, creación, proyectos puntuales ¿Cómo favorece el capital (simbólico, social) acumulado por los portadores de proyectos el alcance y el resultado de las recaudaciones ? ¿ De qué manera se explota la dimensión social de este tipo de procesos de recolecta de fondos ? En este texto, en primer lugar, se discuten los límites de la literatura internacional. Posteriormente se presentan los datos relativos al sector de la información. Finalmente se consideran los aspectos relacionados con la búsqueda de visibilidad. De esta manera se critican algunas ideas preconcebidas y se exponen diversas formas de desigualdad entre los medios de comunicación que utilizan este sistema.

Palabras claves : capital simbólico, capital social, periodismo, crowdfunding, economía de los medios de comunicación. 
Analyse sociologique et économique du financement participatif. Ressorts et critiques dans le cas du journalisme

(2010-2015)

\section{Introduction}

Le crowdfunding, traduit usuellement et imparfaitement par les expressions «financement participatif » ou « financement par la foule » (voire par le syntagme sociofinancement), est un procédé de récolte de fonds qui revisite sous une nouvelle forme, depuis la fin des années 2000, le principe de la donation. Précisons d'emblée que nous n'emploierons pas les termes crowdfunding ou financement par la foule pour ne pas créditer l'idée d'une participation massive. De même, les termes don et donateur ne seront pas employés puisque les pratiques relèvent plus de la transaction que du don.

II sera question ici du financement participatif qui fonctionne sur le «don " avec contreparties (rewards-based). II existe d'autres variantes, notamment celle qui concerne le prêt d'argent avec intérêts ou celle qui permet aux contributeurs de prendre une participation au capital d'une entreprise, mais ces deux dernières n'ont pas été utilisées par les médias en France avant $2016^{1}$. De manière générale, une campagne de financement participatif repose sur un principe: récolter un montant d'argent afin de réaliser un projet; et sur deux obligations: financières et temporelles. La plateforme intermédiaire qui reçoit l'argent ne le reverse au porteur d'un projet que si l'objectif financier prédéfini est atteint ou dépassé (100 \% ou plus) à l'issue d'un temps imparti (en moyenne les campagnes durent quarante jours). Les porteurs de projets peuvent librement fixer ce double objectif. En cas d'échec, si le taux de la récolte est inférieur à $100 \%$ à l'issue du temps prévu, tous les contributeurs sont automatiquement remboursés ${ }^{2}$. De leur côté, les plateformes se rémunèrent en prélevant une commission d'environ 5 à $8 \%$ sur les projets finalisés.

Ce type de financement est un phénomène récent qui a gagné en visibilité à l'occasion de collectes symboliques, mais

\footnotetext{
1 Un premier exemple de prise de participation, hors de notre périmètre d'étude (2010 - été 2015), est à signaler du côté du média Les Jours au printemps 2016 (apport minimum de 1000 euros, objectif final de 750000 euros).

${ }^{2}$ La plupart des grandes plateformes fonctionnent sur ce modèle (« tout ou rien »), mais on trouve également d'autres versions : l'argent est versé même si les $100 \%$ ne sont pas atteints (« tout est pris »), plusieurs objectifs sont fixés en amont, etc.
} 


\section{Guillaume GOASDOUÉ}

pas toujours représentatives ${ }^{3}$. La pratique s'est depuis largement répandue, si bien que tout un chacun a déjà pu être sollicité par une connaissance plus ou moins proche pour contribuer financièrement à un projet artistique, entrepreneurial, humanitaire ou étudiant.

Du côté des médias d'information français ${ }^{4}$, c'est fin 2010 que le premier projet à visée journalistique (Paroles de conflits) lance une campagne de financement participatif et obtient 18000 euros. Le projet hybride (webdocumentaire relatant le périple à vélo d'un journaliste parti sillonner plusieurs pays) est atypique $^{5}$. II contribue à faire de la catégorie " journalisme » un secteur pouvant se prêter au système en vogue du financement participatif, à l'instar des autres industries culturelles (Bennett, Chin et Jones, 2015 ; Matthews, Rouzé et Vachet, 2014).

S'il est peu aisé de définir ce qui relève strictement du journalisme (frontières poreuses, absence d'instance de régulation professionnelle, formation non obligatoire), précisons toutefois que notre terrain concerne les médias qui investissent l'enquête et le reportage, quels que soient les sujets (international, culture, environnement), les registres d'expression (texte, photo, bande dessinée) et les canaux de diffusion (site web, papier). Aussi, en comparaison aux secteurs culturels où l'argent récolté est destiné à la production d'un «produit » fini (album, jeu vidéo, objet, film), les médias d'information (contenu de flux) cherchent avant tout à recruter des abonnés sur le long terme ${ }^{6}$.

La multiplication des campagnes et l'essor de leur visibilité médiatique interviennent en 2014 (Nice Matin, Terra Eco) et 2015 (Society, Les Jours) $^{7}$. Mentionnons enfin trois projets européens qui ont dépassé le million d'euros collectés: De Correspondent aux Pays-Bas en 2013, Krautreporter en

\footnotetext{
${ }^{3}$ Album de musique (Grégoire), série devenue film (Veronica Mars ; Noob), etc.

${ }^{4}$ Voir la présentation des projets en annexe.

${ }^{5}$ La campagne, étendue sur 90 jours, est déclenchée par le producteur alors que le journaliste est déjà sur les routes depuis plusieurs semaines.

${ }^{6}$ Des campagnes proposent même comme contrepartie de devenir " abonné à vie » dès lors que le montant dépasse plusieurs centaines d'euros.

7 Sont exclues les péripéties fiscales des sites Médiapart et Arrêt-sur-images (novembre 2015) à la suite d'un vieux différent avec les services fiscaux (application du "taux super réduit de TVA »). Les deux sites ont lancé en même temps des appels à soutien en s'appuyant sur une plateforme réservée aux médias («j'aime l'info ») - nous y reviendrons -, mais aussi, pour Arrêt-sur-images, sur la plateforme lucrative Ulule.
} 
Analyse sociologique et économique du financement participatif. Ressorts et critiques dans le cas du journalisme

(2010-2015)

Allemagne en 2014 et El Español en Espagne en 2015. Ces trois exemples ont assurément marqué les esprits dans un secteur particulièrement touché sur le plan de l'indépendance ${ }^{8}$ et de l'économie (chute des ressources publicitaires, essor des bloqueurs de publicités en ligne [adblocks], hyperconcurrence, « coopétition » avec l'oligopole de l'internet ${ }^{9}$ ).

Trois hypothèses seront principalement mises en question dans le développement qui suit: le financement participatif n'est-il pas principalement un outil à finalité communicationnelle, et dans une moindre mesure économique, indexé sur le capital symbolique ${ }^{10}$ des porteurs de projets ? Par ailleurs, ce phénomène touche-t-il indistinctement « la foule »? Enfin, ce système n'avantage-t-il pas les porteurs de projets qui ont déjà une expérience médiatique à faire valoir, notamment au sein des médias établis, ce qui rendrait compte d'un principe des avantages cumulés (" effet Matthieu »), lequel signifie que les plus « gros » tirent plus d'avantages d'un système donné ?

Nos résultats, qui ne pourront traiter ici du phénomène dans son ensemble ${ }^{11}$, seront développés en quatre temps. L'évocation de la littérature et de ses carences occupera le premier temps. Nous détaillerons dans un deuxième temps les caractéristiques des campagnes pour le secteur du journalisme en en dégageant les principales tendances. Le troisième temps envisagera plusieurs aspects de la dimension sociale du financement participatif, au regard de la notion de capital (social, symbolique). Dans un quatrième et dernier temps, quelques mécanismes communicationnels qui corroborent la thèse des avantages cumulés seront décrits.

\footnotetext{
${ }^{8}$ Songeons aux nombreux médias (Les Échos, Le Parisien, L'Express, Libération, Le Figaro, Le Monde) dont le capital est majoritairement détenu par des milliardaires (Arnault, Drahi, Dassault, Pinault, Niel, Bolloré).

9 Des sites comme Facebook ou Google sont à la fois des partenaires et des concurrents. Voir, par exemple, Rebillard et Smyrnaios, 2010.

10 Sorte de « capital de reconnaissance » (Bourdieu) - nous développerons par la suite.

${ }^{11}$ Nous nous permettons de renvoyer à notre article (Goasdoue, 2016) qui aborde plus spécifiquement les aspects communicationnels et idéologiques du procédé.
} 


\section{Méthodologie d'enquête}

Notre enquête s'appuie sur plusieurs matériaux dont 16 entretiens, d'une durée de 25 minutes à 2 heures, réalisés en présence ou au téléphone (2014-2015). Ont été interviewés 14 porteurs de projets, après que leurs campagnes soient achevées, et deux responsables des deux principales plateformes généralistes ${ }^{12}$ en activité en France (Ulule et Kisskissbankbank) ${ }^{13}$. Notons que les porteurs de projets interviewés prennent la parole à différents titres sans que cela soit toujours explicité. En effet, en fonction de la taille du média et de la division du travail en interne, les interlocuteurs peuvent avoir plusieurs casquettes (journaliste, start-uper, communicant, actionnaire).

De plus, nous avons effectué une veille en ligne pour suivre la mise en place de campagnes et la progression des collectes, en observant également les discours véhiculés et leur médiatisation (notamment sur les réseaux socionumériques ou lors de journées d'échanges interprofessionnelles). Nous avons en outre suivi les (ré)actions du côté des instances gouvernementales (lois, rapports) ${ }^{14}$ qui visent à réajuster le système des aides publiques à la presse (statuts, avantages fiscaux). Le principe de la donation, que le financement participatif vient en quelque sorte revisiter, est déjà concerné par des dispositifs incitatifs puisque les médias reconnus peuvent appliquer une défiscalisation partielle (pour les particuliers, $66 \%$ du don dans la limite de $20 \%$ des revenus imposables). Un dernier matériau provient des données fournies par les deux principales plateformes (statistiques, bilan annuel, conseils et astuces).

\footnotetext{
12 À l'échelle internationale, les plateformes uniquement dédiées au journalisme ont quasiment toutes disparues. Le projet français Glifpix (inspiré de la plateforme étatsunienne Spot.us) est resté quelques semaines en « beta publique » (fin 2010) avant d'être définitivement abandonné faute d'avoir trouvé un modèle économique.

${ }^{13} \mathrm{La}$ plateforme étatsunienne Kickstarter, lancée en 2009 et comptant parmi les leaders du secteur, arrive en France fin mai 2015.

14 Trois exemples : la loi du 17 avril 2015 créant le statut d' « entreprise solidaire de presse »; l'« amendement Charb " voté au Sénat le 5 février 2015 ; le rapport Charon (Presse et numérique: invention d'un nouvel écosystème) remis le 2 juin 2015 à la ministre de la Culture (Fleur Pellerin).
} 
Analyse sociologique et économique du financement participatif. Ressorts et critiques dans le cas du journalisme

(2010-2015)

\section{Une littérature récente et encore déficiente}

Plusieurs remarques peuvent être énoncées au sujet des travaux qui associent journalisme et financement participatif (Aitamurto, 2011 ; Burtch, Ghose et Wattal, 2013 ; Cagé, 2015 ; Carvajal, Garcia-Aviles et Gonzalez, 2012 ; Jian et Shin, 2014 ; Jian et Usher, 2014 ; Hunter, 2015 ; Sanchez-Gonzales et Palomo-Torres, 2014).

Premièrement, ces études ne portent pas sur les exemples français. Les résultats ne peuvent donc être utilisés qu'après un travail de contextualisation, car les habitudes médiatiques des populations (taux de lecture de la presse, consentement à payer pour s'informer), le taux d'équipement technologique, la pratique ordinaire du don, la place occupée par les fondations, le niveau de l'offre médiatique ou les dispositions légales (défiscalisations, subventions, etc.) différencient les pays et les normes sociales. Concernant le court essai de l'économiste française Julia Cagé (2015), le propos a l'avantage d'inscrire la question des médias dans une réflexion globale qui tente de dépasser le seul prisme économique (politique, gouvernance, démocratie); toutefois, le questionnement du financement participatif ne repose pas sur une étude empirique ni sur la littérature. L'exemple de ce mode de financement vient simplement alimenter une réflexion sur la création d'un nouveau statut (mi-société par actions, mi-fondation) qui reste à l'état théorique.

Deuxièmement, les problématiques soulevées par ces travaux sont hétérogènes, et les focales concernent aussi bien les motivations des "donateurs", les types de sujets financés que les expériences pratiques des journalistes. Ainsi, Hunter (2015) interroge les effets de ce mode de financement sur les marges d'indépendance, d'autonomie et d'objectivité des journalistes. Jian et Usher (2014) comparent les " performances » des journalistes en fonction de leur état de service, pour savoir si les plus expérimentés réussissent mieux leur collecte. Ces deux auteures s'intéressent également aux types de sujets qui sont susceptibles d'être davantage financés, notamment les sujets dits " concernants ${ }^{15}$, ce qui renvoie au paradoxe soulevé par Boczkowski et Peer (2011), lesquels ont

\footnotetext{
15 «[...] stories that would provide [...] practical guidance for daily living (e.g. stories about public health or local city infrastructure) » (Jian et Usher, 2014, p. 164-165).
} 


\section{Guillaume GOASDOUÉ}

constaté que les sujets privilégiés par les consommateurs d'informations ne sont pas forcément ceux que les journalistes affectionnent. Jian et Shin (2014) répertorient les différentes motivations qui animent les contributeurs. Burtch, Ghose et Wattal (2013) orientent leur travail sur la corrélation positive entre l'investissement en matière de marketing et le succès des campagnes. Sanchez-Gonzalez et Palomo-Torres (2014) interrogent quant à elles le degré de familiarité des (étudiants)journalistes avec ce système de collecte de fonds. Enfin, Cagé (2015) articule les questions de financement participatif et les types de gouvernance, avec l'idée que toute transaction effectuée pourrait ouvrir des droits de participation au capital de l'entreprise.

Troisièmement, de multiples ancrages disciplinaires et théoriques alimentent les articles avec des grilles de lecture et des résultats qui ne convergent pas nécessairement. En résumé, la question du crowdfunding a été principalement investie par les sciences de gestion et les sciences économiques, puis par des courants qui s'intéressent parfois moins aux médias, mais davantage aux jeux économiques (incentives). La plupart des chercheurs tendent également à adopter une approche fonctionnaliste qui promeut la figure d'un usager actif ${ }^{16}$. En outre, différents outillages théoriques se font concurrence et le principe de "cumulativité " des connaissances n'est pas toujours appliqué - fut-il applicable si l'on considère les limites que comportent certains travaux. De multiples théories et approches sont ainsi convoquées : interaction homme-machine (Gerber, Hui et Kuo, 2012), usages et gratifications (Jian et Usher, 2014), consumer choice theory (Burtch, Ghose et Wattal, 2013 ; Jian et Usher, 2014), action collective (Jian et Shin, 2014), Willingness to Pay (Goyanes, 2014).

Notons par ailleurs que plusieurs études portent sur le même site étatsunien Spot.us ${ }^{17}$, lequel n'est pas une plateforme

\footnotetext{
${ }^{16}$ On trouvera des critiques de cette inclination dans Matthews, Rouzé et Vachet (2014), dans le sillage d'un premier ouvrage déjà critique à propos du « web collaboratif » (Bouquillion et Matthews, 2010).

17 Aitamurto (2011), Jian et Usher (2014), Jian et Shin (2014). Cela est sans doute dû au fait que le site a ouvert l'accès à ses données aux chercheurs. De plus, cette plateforme fonctionne avec des sondages qui, une fois complétés, sont convertis en crédits que les répondants peuvent utiliser pour soutenir des projets. Des chercheurs ont donc pu, par ce moyen, proposer leur propre sondage au sein de la plateforme.
} 
Analyse sociologique et économique du financement participatif. Ressorts et critiques dans le cas du journalisme

(2010-2015)

généraliste, mais un site de petite taille dédié aux enquêtes journalistiques «citoyennes » et hyperlocales (principalement pour la côte sud-ouest des États-Unis) ${ }^{18}$. Enfin, signalons des limites et des biais méthodologiques (échantillons non représentatifs, rétribution financière des sondés, rationalisme économique) qui viennent s'ajouter aux autres points évoqués ci-dessus ${ }^{19}$. Pour toutes ces raisons, et comme c'est souvent le cas pour tout phénomène émergeant, les connaissances restent encore parcellaires. Un consensus se dégage toutefois autour de quelques observations que nous détaillerons par la suite (poids des ressources préexistantes, modèle de courbe en « $U$ » pour le rythme des collectes, effets positifs du levier mercatique).

De notre côté, nous aborderons le phénomène en recourant au concept de capital (Bourdieu, 1980). En effet, penser en termes de capital (notamment social et symbolique) permet de rendre compte des inégales ressources et de leurs effets sur les rétributions attendues dans le cadre des opérations de financement participatif. La possession d'un capital social ${ }^{20}$ fourni, lequel fait système avec les autres sortes de capitaux (notamment économique et symbolique), accentue potentiellement un effet multiplicateur, ce qui est avantageux dans l'optique de récolter des fonds. Partant, l'exemple du financement participatif permet de pointer la capacité différenciée des journalistes ou des titres médiatiques, à user de leur capital symbolique (en tant que crédit) ${ }^{21}$ pour alimenter leur capital économique.

\footnotetext{
${ }^{18}$ Le site Spot.us a été racheté en 2011 (par le groupe American Public Media), puis a décliné jusqu'à sa fermeture en février 2015.

19 La première chercheure (Aitamurto) qui publie sur le journalisme et le crowdfunding a plusieurs casquettes : universitaire, experte-journaliste, consultante. Cette dernière promeut un journalisme financé par des dons sur le modèle du " cause marketing " (projets charitables et non lucratifs, voués au changement social).

${ }^{20}$ "Le capital social est l'ensemble des ressources actuelles ou potentielles qui sont liées à la possession d'un réseau durable de relations plus ou moins institutionnalisées d'interconnaissance et d'inter-reconnaissance; ou, en d'autres termes, à l'appartenance à un groupe, comme ensemble d'agents qui ne sont pas seulement dotés de propriétés communes (susceptibles d'être perçues par l'observateur, par les autres ou par eux-mêmes) mais sont aussi unis par des liaisons permanentes et utiles »(Bourdieu, 1980, p. 2).

21 «Le capital symbolique [...] n'est pas une espèce particulière de capital mais ce que devient toute espèce de capital, lorsqu'elle est méconnue en tant que capital, c'est-à-dire en tant que force, pouvoir ou capacité d'exploitation (actuelle ou potentielle), donc reconnue comme légitime» (Bourdieu, 1997, p. 283). En
} 


\section{Guillaume GOASDOUÉ}

À la différence de la plupart des travaux associant crowdfunding et journalisme, nous ne chercherons pas à tenir pour acquis ce phénomène, en étudiant isolément l'une de ses facettes ou en proposant des pistes pour en améliorer les mécanismes, mais nous essaierons de le rendre intelligible au moyen d'un appareil critique et d'une étude de cas de première main.

\section{Cas de figure du financement participatif et journalisme}

\subsection{Typologie des campagnes de collecte}

La trentaine de cas étudiés (2010-2015) pour les campagnes relevant du journalisme permet de distinguer quatre principaux cas de figure, lesquels ne sont pas nécessairement exclusifs : la création, le sauvetage, la diversification, le coup-par-coup. Le recours au financement participatif pour une création $\left(1^{\mathrm{er}}\right.$ cas) concerne les nouveaux médias qui cherchent un appui financier pour développer un site internet, pour lancer une enquête, pour acheter du matériel (Hexagones, Les Jours) ou pour constituer, via le système du préabonnement, une communauté de bêtatesteurs (Brief.me). Pour le sauvetage $\left(2^{\mathrm{e}}\right.$ cas), on a affaire aux médias en mauvaise posture qui tentent de renflouer leur trésorerie pour survivre ou se relancer sur de nouvelles bases (Nice Matin, Terra Eco, Rue89-Strasbourg, Marsactu, Courrier des Balkans, Aqui). La diversification ( $3^{\mathrm{e}}$ cas) induit le développement d'un projet sur un nouveau support (papier, internet, audiovisuel) ou une nouvelle thématique (La France vue d'ici, Society, Hors-Série). Enfin, le financement en mode " coup-par-coup " ( $4^{\mathrm{e}}$ cas) implique de recourir régulièrement au financement participatif. Le mécanisme est structurel et a vocation à être répété (Enquête ouverte). II s'agit par exemple de proposer des thèmes d'enquête qui ne sont réalisés qu'une fois les fonds réunis. Un autre exemple concerne les projets qui

complément: le capital symbolique est « un capital de reconnaissance qui a sa logique propre d'accumulation, de conservation, de transmission et aussi de conversion en d'autres espèces de capital » (Bourdieu, 2015, p. 134). 
Analyse sociologique et économique du financement participatif. Ressorts et critiques dans le cas du journalisme

(2010-2015)

font appel aux consommateurs au début de chaque nouvelle saison (La France vue d'ici, Kaïzen) ${ }^{22}$.

On le pressent, les projets dont il est question sont composites et ne se prêtent pas aisément à l'exercice de la classification. Des projets peuvent correspondre à deux catégories au même moment ou au fil de leur évolution. HorsSérie est à la fois un nouveau média produisant des entretiens vidéo avec des intellectuels et des artistes, mais il peut également être considéré comme étant un projet de diversification émanant du média Arrêt-sur-images avec lequel il est fortement lié (moyens techniques, ressources humaines, abonnements croisés). La création de ces sous-groupes fait office d'outil descriptif (provisoire). II ne s'agit pas de catégories analytiques en tant que telles, mais plutôt d'une présentation qui vise à retranscrire la complexité de l'objet observé, notamment par souci de comparaison avec d'autres secteurs des industries culturelles. Cette phase descriptive se veut également un préalable pour nuancer les idées reçues et apporter des données concrètes que chacun pourra librement apprécier. Enfin, pour être complet, rappelons que la recherche de fonds peut passer par d'autres canaux (actionnaires, abonnements directs, subventions, fonds d'aide, annonceurs, partenariats, formation), mais également par des plateformes non lucratives dédiées spécifiquement aux médias d'information - nous y reviendrons.

\subsection{Données chiffrées : campagnes et plateformes}

Les ordres de grandeur (sommes collectées, ratio entre le montant espéré et le montant obtenu, nombre de contributions $^{23}$ ) sont contrastés ${ }^{24}$. En 2015, la collecte la plus importante pour le cas du journalisme en France est celle menée par une équipe de salariés du quotidien Nice Matin $\left(376000\right.$ euros $\left.^{25}\right)$. Ces derniers avaient pour objectif de

\footnotetext{
22 C'est le cas, aussi, de Gonzaï, un magazine culturel qui a récolté 26665 euros (décembre 2014, 547 contributions) afin de financer l'année 2015.

${ }^{23}$ Les données accessibles sur les plateformes ne permettent pas de connaître le nombre de contributeurs, mais seulement le nombre de contributions.

${ }^{24}$ Nous ne nous sommes pas intéressé aux campagnes modestes de quelques centaines d'euros.

${ }^{25}$ Pour information, le record français toute catégorie est détenu par Noob, le projet d'adaptation d'une websérie amateur en film (fin de collecte le 7 décembre 2013 , 681000 euros, 11930 contributions) (http://fr.ulule.com/noob-le-film/).
} 


\section{Guillaume GOASDOUÉ}

racheter le titre régional après que celui-ci ait été placé en redressement judiciaire. Concernant les pourcentages - qui n'ont de sens qu'après contextualisation -, soit la somme atteinte rapportée à la somme souhaitée, les chiffres vont de $35 \%\left(A q u i^{26}\right)$ à $795 \%$ (Society). Si l'on met de côté le cas atypique de la collecte du site régional Aqui, on observe que tous les médias franchissent le cap des $100 \%$, ce qui invite à interroger les fondements d'un "jeu » où $100 \%$ des participants remportent leur pari - en référence au slogan dont usait en son temps la loterie nationale : « $100 \%$ des gagnants ont tenté leur chance $»$.

Le nombre maximum de contributions pour une campagne est de 3188 pour le média Hors-Série. Enfin, l'échelle des montants donnant droit à des contreparties de plus en plus conséquentes - bien que parfois symboliques (nom crédité, possibilité d'assister à une conférence de rédaction)s'échelonne de cinq à plusieurs milliers d'euros ${ }^{27}$.

S'agissant des données chiffrées des deux principales plateformes généralistes qui hébergent la quasi-totalité des projets journalistiques en France ${ }^{28}$ (Tableau 1), on obtient, pour le site Ulule, une catégorie "Journalisme \& édition", qui se situe en $4^{\mathrm{e}}$ position (sur 15) en matière de montants collectés ${ }^{29}$, soit, sur la période 2010 à juillet 2015, un total de 2,9 millions d'euros. Cela correspond à 71861 contributions, pour 482 campagnes réussies sur un total de 773 (62\%), avec un « don » moyen de 43 euros. Pour le site Kisskissbankbank, la catégorie "journalisme ", en $11^{\mathrm{e}}$ position sur les 17 catégories $^{30}$, affiche 1,2 million d'euros collectés pour la même période (à quelques mois près puisque le site était ouvert avant son concurrent susmentionné). Dans l'ensemble, 199 campagnes ont été réussies sur un total de 362 (soit un

\footnotetext{
${ }^{26}$ La collecte du site régional Aqui a une triple particularité. Premièrement, le taux final $(35 \%)$ est sous la barre des $100 \%$. Deuxièmement, le site est passé par un service de plateforme personnalisable (Mipise.com). Troisièmement, cette plateforme repose sur le principe du « tout est pris » (soit, pour Aqui : 31885 euros sur l'objectif initial des 90 000).

${ }_{27}$ Les contributeurs et contributrices ont la possibilité, en cochant une case, de renoncer aux contreparties.

${ }^{28}$ Les données proviennent des deux plateformes.

29 Les premières catégories sont: "Film et vidéo », " Musique », " Solidaire et citoyen $»$.

30 Les premières catégories sont: « Musique », "Film et vidéo », « Spectacle », « Art vivant », « Solidarité ».
} 
Analyse sociologique et économique du financement participatif. Ressorts et critiques dans le cas du journalisme

(2010-2015)

taux de réussite de $55 \%$ ). Enfin, 24716 contributions ont été enregistrées avec un « don » moyen de 55 euros.

Tableau 1 - Catégorie « journalisme » sur les deux principales plateformes

\begin{tabular}{|c|c|c|}
\hline $2010-07 / 2015$ & Ulule & KissKissBankBank \\
\hline Catégorie & $\begin{array}{l}\text { "Journalisme et } \\
\text { édition » }(4 / 15)\end{array}$ & $\begin{array}{c}\text { "journalisme » } \\
(11 / 17)\end{array}$ \\
\hline $\begin{array}{l}\text { Montants reversés } \\
\text { (millions d'euros) }\end{array}$ & 2,9 & 1,2 \\
\hline $\begin{array}{l}\text { Nombre de } \\
\text { contributions }\end{array}$ & 71861 & 24716 \\
\hline $\begin{array}{l}\text { Taux de réussite } \\
\text { (projets) }\end{array}$ & $\begin{array}{c}62 \% \\
(482 / 773)\end{array}$ & $\begin{array}{c}55 \% \\
(199 / 362)\end{array}$ \\
\hline Don moyen (euros) & 43 & 55 \\
\hline
\end{tabular}

Si les deux sites proposent sensiblement les mêmes services et prélèvent les mêmes taux de commission, Ulule héberge cependant plus de projets dans la catégorie "journalisme ». II faut sans doute y voir comme principale raison le rôle clé, à partir de septembre 2012, du directeur des projets et de la communication. En effet, ce dernier a été responsable du développement numérique et rédacteur en chef adjoint du site web d'un hebdomadaire (Marianne). Cet ancien journaliste est également très présent en ligne par le truchement de comptes sur les réseaux socionumériques (Twitter). De nombreux porteurs de projets nous ont ainsi expliqué connaître (in)directement cet intermédiaire ${ }^{31}$ qui a le double avantage d'être au fait de la situation des médias d'information ${ }^{32}$ et de disposer encore d'un carnet de contacts auprès des rédactions, ce qui peut s'avérer utile comme nous le verrons dans la dernière partie.

\footnotetext{
${ }^{31}$ Extrait d'entretien : "On se dit on va faire du crowdfunding. On en connait deux [plateformes], Ulule et KissKissBankBank. On regarde un peu dans nos répertoires, bon, ça va vite. On a quelques contacts. Le premier contact, c'était $\mathrm{M}$. de Ulule, qui est un ancien journaliste de Marianne, et qu'on connaissait comme ça. Donc on l'appelle » (Journaliste de Nice Matin).

32 Deux cofondateurs d'Ulule ont également travaillé dans le journalisme culturel.
} 


\section{Guillaume GOASDOUÉ}

\section{Ressorts sociaux du financement participatif}

Au premier abord, le rôle économique et le caractère massif de ce mode de récolte de fonds semblent essentiels, comme en attestent le terme financement (funding) et le préfixe foule (crowd). Or, en matière économique, d'une part, on observe des exemples contrastés. Par exemple, la collecte des salariés de Nice Matin (376 000 euros) correspond à environ $2 \%$ du montage financier (16 millions) que ces derniers ont constitué afin de convaincre le tribunal dans leur projet de reprise du journal. En revanche, les 70000 euros récoltés par Hors-série représentent la quasi-totalité des ressources pour la première année d'exercice. Dans la majeure partie des cas, cependant, les apports des collectes recouvrent une faible part de l'ensemble des dépenses des médias. D'autre part, le terme foule n'est vraisemblablement pas le qualificatif le plus approprié pour décrire le phénomène. Les projets étudiés plafonnent en moyenne à quelques centaines de contributions (jusqu'à 3188 pour Hors-série), alors que ces mêmes médias, du moins ceux qui existaient déjà avant le lancement d'une collecte, revendiquent des dizaines de milliers, voire des centaines de milliers de lecteurs.

\subsection{Ancrages du phénomène}

Le recours au financement participatif dans le cas du journalisme peut être rattaché à plusieurs phénomènes plus anciens qui vont du crowdsourcing ${ }^{33}$ au «Web $2.0^{34}$ ", avec l'essor des contenus générés par les usagers (User-Generated Content $)^{35}$, en passant par le développement plus récent des réseaux socionumériques. Le partage d'un socle commun, aux racines parfois très anciennes, peut être également évoqué avec différentes formes de mécénat ou de pratiques altruistes :

\footnotetext{
33 «La production participative est un modèle de production et de résolution de problèmes distribués en ligne " (traduction libre de: "Crowdsourcing is an online, distributed problem-solving and production model ») (Brabham, 2008, p. 75).

34 Terme qui aurait été forgé par Darcy DiNucci (1999), mais dont la popularité, à partir de 2004, est associée aux interventions de Tim O'Reilly et de son entourage (voir, par exemple, les sources répertoriées dans la version anglaise de l'encyclopédie Wikipedia).

${ }^{35}$ En 2006, le magazine Time désigne comme personne de l'année « You », c'est-àdire les millions d'internautes anonymes qui contribuent d'une manière ou d'une autre à créer-diffuser-signaler des contenus.
} 
Analyse sociologique et économique du financement participatif. Ressorts et critiques dans le cas du journalisme

(2010-2015)

évergétisme ${ }^{36}$, dons, opérations de type fundraising (humanitaire, politique, charité). Plus particulièrement, songeons encore au système de souscription dans les domaines artistique ${ }^{37}$ ou journalistique. En effet, les médias en mauvaise posture financière ont toujours fait appel à leur lectorat. En juin 2015, après plusieurs semaines d'appel aux dons, le quotidien L'Humanité annonçait avoir reçu 1,8 million d'euros de la part d'environ 13000 personnes (23 juin 2015, p. 8). Pour l'histoire, le journal créé en 1904 lance sa première souscription dès 1907. Autre point de comparaison, le mensuel Le Monde diplomatique, qui sollicite régulièrement ses lecteurs, a obtenu 242000 euros de dons en 2013 et 296000 euros en 2014 ( $n^{\circ} 739$, octobre 2015). Ces deux médias ont fait transiter les dons via "Presse et Pluralisme », une des deux plateformes destinées aux publications d'information politique et générale $(I P G)^{38}$. Ce service, créé fin 2007 à l'initiative de plusieurs syndicats de la presse et hébergé en ligne par la Caisse des Dépôts, ainsi que l'autre plateforme, "J'aime l'info ", créée en mars 2011 et chapeautée depuis plusieurs années par le Syndicat de la presse indépendante d'information en ligne (SPIIL), permettent l'application de défiscalisations. Aussi, contrairement aux plateformes lucratives, les dons sont reçus à longueur d'année sans que ne soient déterminés une période arrêtée et un seuil minimal à atteindre.

Le financement participatif fraie également avec le monde de l'innovation appliquée au domaine économique et politique. David Cohn ${ }^{39}$, fondateur de la plateforme étatsunienne Spot.us, a coutume d'expliquer que l'inspiration lui est notamment venue des micro-donations enregistrées dans le camp Obama lors de la présidentielle de 2008.

Si le financement participatif connaît une forte expansion et un accueil favorable - les critiques restent rares -, il semble

\footnotetext{
${ }^{36}$ Dons à la collectivité offerts par les notables et les sénateurs de l'époque antique. Voir « Le pain et le cirque » (Veyne, 1976).

37 Souscriptions pour des ouvrages (Jane Austen, Mark Twain) ou des films (Shadows de John Cassavetes, 1959) (exemples cités dans Matthews, Rouzé et Vachet, 2014).

${ }^{38}$ Une vingtaine de médias utilisent ce service en 2015: Charlie Hebdo, Politis, Causette, Alternatives économiques, La Marseillaise, Books, etc.

${ }^{39}$ Voir, par exemple : KERSHAW S., 2008, 23 août, « A Different Way to Pay for the News You Want », Sarah Kershaw, The New York Times,

<http://www.nytimes.com/2008/08/24/weekinreview/24kershaw.html>, dernière consultation le 28 février 2017.
} 


\section{Guillaume GOASDOUÉ}

pourtant que des limites apparaissent, notamment si l'on se réfère aux travaux qui pointent certains revers de l'économie numérique (digital labor ${ }^{40}$, "solutionnisme technologique ${ }^{41}$ ", "ferme de contenus ${ }^{42}$ ", "mise au travail des consommateurs $\left.{ }^{43} »\right)$. De fait, en participant aux campagnes, les individus effectuent plus que de simples "dons", puisqu'ils prennent également en charge une partie du travail des médias, des banques, de l'État et des communicants. De plus, toutes les activités créent mécaniquement des (méta)données qui constituent une valeur d'usage pour les entreprises de l'internet (production de statistiques, ajustement d'algorithmes de personnalisation et de recommandation, création de patterns), sans qu'il y ait de rétribution équitable en échange.

Ce mode de financement, tel qu'il est porté par les plateformes lucratives, s'apparente in fine à une reconfiguration, sous de nouveaux atours (numériques), de la relation économique entre des porteurs de projets et des contributeurs, relation qui est également qualifiée par des chercheurs et des interviewés par les expressions: "social funding ", "friend funding", "love money". Autant de qualificatifs qui témoignent du caractère éminemment social de la pratique, laquelle repose sur des réseaux et des communautés pour une grande partie préconstitués, d'où l'intérêt de mobiliser le concept de capital (social, symbolique) pour en comprendre les ressorts et les effets.

\subsection{De l'ami(-lecteur) au contributeur}

En substance, le discours relayé par les plateformes de financement participatif postule que trois cercles successifs alimentent les collectes: les connaissances du premier niveau

\footnotetext{
40 Travail numérique qui procède des activités des internautes, lesquels créent, en partie à leur insu, de la valeur pour les entreprises qui proposent les services, d'où le rapprochement théorique avec le concept d'exploitation (voir : Cardon et Casilli, 2015 ; Hesmondhalgh, 2010 ; Scholz, 2012).

41 Terme forgé par le chercheur et essayiste Evgeny Morozov (2014) : «Pour tout résoudre, cliquez ici. L'aberration du solutionnisme technologique " (To Save Everything. Click Here, 2013).

${ }^{42}$ Content Farm ou Click Farm : site web dont l'objectif est de produire beaucoup de contenus à bas coûts pour être bien référencé et toucher des revenus publicitaires substantiels (voir, par exemple : Bakker, 2012, «Aggregation, Content Farms and Huffinization ").

${ }^{43}$ Dujarier, 2008.
} 
Analyse sociologique et économique du financement participatif. Ressorts et critiques dans le cas du journalisme

(2010-2015)

(famille, amis, collègues), les relations des connaissances du premier cercle (amis d'amis) et le grand public. Un deuxième précepte explique que, pour susciter des contributions et créer une dynamique de confiance susceptible de se propager, une campagne ne doit pas partir de (presque) rien au moment de son lancement public. Dans le cas d'une création de médias, les responsables des plateformes énoncent, chiffres à l'appui (ceux des moyennes observées), que le porteur de projet doit " amorcer la pompe » en sollicitant en amont des personnes prêtes à dépenser de l'argent dès les premières heures de la collecte $^{44}$. Ces personnes ne sont pas forcément la cible à proprement parler du futur service ou média, mais des connaissances qui, en effectuant une transaction, soutiennent un ami, une aventure ou une démarche jugée courageuse. Plusieurs chercheurs ont d'ailleurs constaté qu'une partie des contributeurs ne venaient pas lire les reportages dont ils avaient financé la production en amont (Aitamurto, 2011; Jian et Usher, 2014). En l'espèce, la démarche a donc parfois moins à voir avec une relation économique basée sur un calcul rationnel (coût économique/bénéfice informationnel), mais semble plutôt être le produit d'un rapport social (don/contre don, estime de soi, altruisme). Un responsable d'une plateforme explique le mécanisme à sa façon :

«Le premier cercle vient valider l'existence du projet [...] ou invalider. C'est-à-dire que si demain vous lancez votre projet [...] si vos amis, votre famille ou vos collègues de travail ne vous suivent pas, c'est quand même qu'à un moment y a un problème, qu'ils n'ont pas confiance. Ils savent que ça ne va pas le faire, etc. Et ça va valider la crédibilité des projets auprès de cercles de plus en plus large. Et plus on est éloignés et plus on a besoin de gens qui viennent valider cette crédibilité du projet. ॥

Cette méthode est aussi résumée par une journaliste qui a récolté plusieurs milliers d'euros :

«On a été voir les familles et amis [...] tout commence par le cercle proche, et après on élargit, on élargit, et on essaie d'arriver aux gens qu'on ne connaît pas. Mais on ne peut pas convaincre les gens qu'on ne connaît pas, direct avec une caisse vide. Donc il faut commencer par faire un fonds de

\footnotetext{
${ }^{44}$ Dans le cas des médias connus qui se relancent ou se diversifient, cette logique est moins décisive.
} 


\section{Guillaume GOASDOUÉ}

caisse avec les gens autour, ça c'est un premier truc. Ensuite on attaque des gens plus concernés, etc. "

Les récits convergent sur ce point et indiquent qu'il faut avoir l'assurance que les proches peuvent alimenter le lancement de la campagne et/ou assurer le succès du projet dans le temps imparti, particulièrement dans les derniers jours, voire les dernières heures ${ }^{45}$. Les détenteurs d'un capital social bien pourvu disposent ainsi d'un avantage manifeste pour fixer à la hausse le seuil à atteindre et pour limiter les risques quant à leur capacité à lever des fonds. Des porteurs de projets interviewés ont évoqué une tante aux " poches sans fond " ou des " copains prêts à sortir leur carte bancaire " pour donner (parfois une deuxième fois en cas de besoin). De fait, les courbes de progression des collectes tendent à dessiner un modèle en " $U$ », avec beaucoup de dons en début et en fin de campagne. Autre constat, un tassement du rythme des transactions est habituellement constaté dès que l'objectif $(100 \%)$ est franchi.

Les campagnes qui respectent le mieux ces principes optimisent plus facilement leur collecte. Partant, on observe un degré de professionnalisation croissant parmi les projets les plus récents qui acceptent d'investir les techniques mercatiques et qui y consacrent des moyens (ressources humaines, réalisation de vidéos de bonne facture, animation permanente sur les canaux de communication, organisation d'événements, relations publiques). Par exemple, au moment où le futur média Les Jours lance sa communication à destination du grand public via une newsletter et des messages publiés sur les réseaux socionumériques, la collecte affiche déjà $20 \%$ de l'objectif, soit 10000 euros récoltés sur les 50000 espérés. Concrètement, la page web avait été créée la veille et les premiers réseaux avaient été sollicités dans la foulée.

Toutefois, malgré l'expérience accumulée et la rationalisation à l'œuvre dans la gestion des campagnes ${ }^{46}$, il reste une part d'inconnus que les porteurs de projets regrettent de ne pouvoir mieux maîtriser : comment fixer un montant qui ne soit ni trop

\footnotetext{
45 Rue89-Strasbourg a entamé sa dernière journée de collecte à $68 \%$ (23 800 euros), avant de clôturer la campagne quelques heures plus tard à $103 \%$ (36 395 euros).

46 II est aussi conseillé aux futurs porteurs de projets de se socialiser par eux-mêmes au système en contribuant à d'autres campagnes.
} 
Analyse sociologique et économique du financement participatif. Ressorts et critiques dans le cas du journalisme

(2010-2015)

élevé, au risque de tout perdre si les $100 \%$ ne sont pas atteints, ni trop modeste, au risque de ne pas optimiser l'opération (car une fois l'objectif franchi, le rythme des contributions tend à s'essouffler) ? Et comment motiver les contributeurs quand la dimension économique apparaît souvent très secondaire ${ }^{47}$ ? De surcroît, comment susciter des transactions, alors que le principe du financement participatif concerne de plus en plus de domaines (petites entreprises, associations, patrimoine, sport, humanitaire, art, santé, etc.), rendant la concurrence entre les projets de plus en plus vive ?

On comprend donc que les médias et les journalistes qui disposent d'un capital symbolique conséquent bénéficient d'un avantage non négligeable au sein d'un champ médiatique fortement concurrentiel.

\subsection{Les plateformes: un dispositif indexé sur les interactions sociales}

Le caractère social du crowdfunding se manifeste également par la conception et l'inscription de l'activité au sein de l'écosystème numérique et des réseaux de sociabilités ${ }^{48}$. Les plateformes et les campagnes sont pensées et conçues de sorte que les interactions sociales soient en partie prescrites par le dispositif. La plateforme opère comme un réseau social qui tisse des passerelles avec les réseaux les plus dominants (Facebook, Twitter). II est ainsi possible, une fois qu'un compte est créé sur la plateforme, de suivre l'actualité des contributeurs et des porteurs de projets de son choix. II est aussi prévu que les traces des activités (transactions) et les pages dédiées aux campagnes puissent être relayées facilement, en écho à la tendance plus générale de l'«économie de la

\footnotetext{
${ }^{47}$ Le magazine Society était dans les kiosques cinq jours après la fin de la collecte. Celle-ci avait donc peu d'incidence sur le lancement du quinzomadaire. Par ailleurs, le montant demandé était faible (6315 euros en référence à la date de sortie du $1^{\mathrm{er}}$ numéro : 6/3/15) pour un groupe de presse installé (SoFoot, SoFoot Club, SoFilm, Doolittle, Pédale) dont la prévision du chiffre d'affaire de 2015 dépassait les 10 millions d'euros. On le voit ici, le financement participatif a parfois d'autres fonctions, comme la communication ou la constitution d'une base d'abonnés à partir du système de préabonnement.

${ }^{48}$ En référence aux travaux mésosociologiques sur les réseaux sociaux, dont on trouvera une synthèse chez Mercklé (2016), le financement participatif pourrait être approché comme un système qui vise à exploiter les "cliques ", les "trous structuraux " ou, pour reprendre la formule de Granovetter, la «force des liens faibles » qui relient les agents sociaux avec différents degrés.
} 


\section{Guillaume GOASDOUÉ}

recommandation " et de la "like economy ${ }^{49}$ ". Les relais s'effectuent par des messages préformatés que les usagers peuvent publier sur les réseaux socionumériques ou recevoir dans leur messagerie chaque fois que leurs connaissances effectuent une contribution (« untel vient de participer à tel projet $»)$.

De plus, les plateformes bénéficient des interactions sociales qui se font jour entre les porteurs de projets et les particuliers qui, dans une logique maussienne de réciprocité (don/contredon), nouent des relations. Plusieurs journalistes nous ont ainsi expliqué qu'ils participaient aux projets de leurs confrères, en espérant, d'une part, se faire connaître d'eux et en misant, d'autre part, sur le fait que ces derniers participent en retour au financement de leur propre campagne ou deviennent des relais de communication auprès de leurs réseaux sociaux et professionnels respectifs - aspect qui peut être décisif pour une population professionnelle qui a fortement investi les réseaux socionumériques (Artwick, 2013 ; Bruns, 2012 ; Lasorsa, Lewis et Holton, 2012; Vis, 2013). Ce phénomène est particulièrement visible dans le cas du projet Les Jours (collecte achevée le 13 juillet 2015) dont le lancement du site, par des anciens de Libération, était initialement prévu fin 2015. En effet, beaucoup de journalistes, de différents horizons, ont contribué et publié un message de soutien sur les réseaux socionumériques comme Edwy Plenel et Fabrice Arfi de Médiapart, lesquels ont plus de 500000 suiveurs sur le réseau Twitter, pour le premier, et plus de 70000 suiveurs pour le second $^{50}$. Cette logique interprofessionnelle de soutiens croisés est encore plus forte sur les plateformes spécialisées (en journalisme), comme a pu le remarquer Aitamurto (2011) dans le cas de l'ancien site Spot.us, où beaucoup de contributeurs étaient en fait des journalistes.

Du côté des plateformes, celles-ci opèrent comme des intermédiaires, intercalés entre les producteurs et les consommateurs d'informations, dont le modèle d'affaires est

\footnotetext{
49 Gerlitz et Helmond (2013) ont forgé cette expression à propos de Facebook pour qualifier la logique d'expansion de la firme sur le web, dans l'optique de capter des données par l'implémentation de boutons (like, share) et de cookies sur des sites tiers.

${ }^{50}$ Nombres très élevés par rapport à ceux que l'on observe chez les journalistes. Toutefois, ces chiffres - qui ne disent rien en matière de réception - sont à prendre avec du recul (faux comptes, comptes peu actifs, robots, etc.).
} 
Analyse sociologique et économique du financement participatif. Ressorts et critiques dans le cas du journalisme

(2010-2015)

basé sur le prélèvement de commissions (5 à $8 \%$ ). Elles ont donc tout intérêt à se faire connaître et à héberger de plus en plus de projets prometteurs ${ }^{51}$. En effet, une fois le service développé, les coûts d'exploitation sont marginaux. De plus, comme c'est souvent le cas dans l'économie numérique, particulièrement avec des services peu différenciés, la forte concurrence induit des situations où peu d'acteurs survivent, en l'occurrence tous les projets portés par des journalistes en France l'ont été très majoritairement sur deux plateformes (Kisskissbankbank et Ulule).

\section{Recherche de visibilité et poids des relais médiatiques}

Plusieurs leviers sont activés par les médias qui usent de leur capital symbolique pour accroître leur capital économique. Un des résultats escomptés est, par exemple, de toucher le grand public par le truchement de personnes influentes ou de médias installés. Ainsi, pour intéresser les journalistes (gatekeepers) et pour bénéficier du rayonnement des titres médiatiques susceptibles de toucher des dizaines ou des centaines de milliers de lecteurs, les porteurs de projets peuvent compter sur l'association opérée entre leur identité et une figure ou une marque déjà connue. Hors-Série bénéficie du soutien du média Arrêt-sur-Images ; La France vue d'ici mise sur le partenariat avec Mediapart et le festival photographique de Sète; Hexagones met en avant l'amitié nouée avec le journaliste Denis Robert (« affaire Clearstream»); Kaïzen s'appuie sur une personnalité (Pierre Rabhi) et une communauté (« mouvement colibris ») $)^{52}$; Les Jours comptent principalement sur leur expérience passée au sein de Libération; Rue89-Strasbourg joue implicitement sur la « marque » Rue $89^{53}$.

\footnotetext{
${ }^{51}$ Sont exclus les projets jugés insensés afin de ne pas entamer le taux de réussite moyen des campagnes, lequel indicateur (environ $60 \%$ de réussite pour la catégorie « journalisme ») sert d'argument commercial face aux concurrents pour attirer les porteurs de projets.

52 Pierre Rabhi, essayiste et agriculteur, est à l'origine du " mouvement colibris », organisation visant à promouvoir un " projet de société écologique et humain ».

53 Un simple accord autorise le site régional à employer le nom et la charte graphique de Rue89, mais les rédactions et les sociétés sont indépendantes.
} 


\section{Guillaume GOASDOUÉ}

Les campagnes de financement participatif recourent également aux techniques de communication, lesquelles se déclinent avec du storytelling (l'art de mettre en récit une histoire $)^{54}$, la publication tous azimuts sur les réseaux socionumériques ou la sollicitation active de relais médiatiques qui seront à même d'animer et de faire exister une campagne. La responsable du site Hors-Série confirme la corrélation souvent instantanée entre le rythme de la collecte et les messages véhiculés par des personnalités : "À chaque fois que Daniel Schneidermann [Arrêt sur images] lançait une information sur le fait qu'on était en train de faire du crowdfunding, on avait tout d'un coup une courbe ascendante de souscriptions. C'était très spectaculaire. »

Un journaliste de Nice Matin insiste sur l'importance de mobiliser l'ensemble des canaux de diffusion, et ce, sans relâche : «La clé, ça a été la com[munication]. On avait créé une page Facebook où on relayait nos messages. On avait créé un Tumblr. On a inondé Twitter avec nos comptes perso [...] On a essayé de faire vivre au maximum le truc sur les réseaux sociaux. "

La démarche est la même du côté de Rue89-Strasbourg, où l'ensemble des espaces publicitaires des pages du site (bandeaux, habillage) a été investi pour promouvoir la campagne. Chez Mars-actu (Marseille), un «kit de communication " comprenant des visuels préformatés (dimensions et messages) a été mis à disposition afin que les internautes personnalisent leurs comptes sur les réseaux. On a affaire, ici, à ce que Singer (2014) qualifie de "visibilité générée par les utilisateurs " (User-Generated Visibility) dans un contexte où les messages sont disséminés et relayés sur toutes sortes de médias, dont les réseaux socionumériques.

Chaque projet journalistique, en fonction de la cible visée, tente de solliciter les meilleurs intermédiaires, ceux qui sont à même de propager l'information pour convertir des " affinités électives " (Max Weber) en actes concrets (abonnement, contribution, recommandation). Les blogueurs ou «twittos ${ }^{55}$ » influents dans un secteur (journalisme, jeu vidéo, logiciels libres, écologie, etc.) peuvent avoir également beaucoup de

\footnotetext{
${ }^{54}$ Un exemple parmi d'autres : l'anecdote du premier chèque reçu par Nice Matin celui d'une nonagénaire (Simonne P.), qui a donné lieu à des reportages télévisés diffusés dans les journaux de TF1 et France 2.

${ }^{55}$ Détenteurs d'un compte sur le réseau Twitter.
} 
Analyse sociologique et économique du financement participatif. Ressorts et critiques dans le cas du journalisme

(2010-2015)

poids. De même, dans le cas des médias régionaux, le « capital territorial » (Guidici, Guerini et Rossi-Lamastra, 2013) est aussi primordial pour toucher des interlocuteurs de choix comme les décrochages régionaux du journal télévisé de France 3 et de la radio France Bleu.

À ce «jeu », les journalistes disposent d'un avantage comparatif puisqu'ils font partie, à divers degrés, d'un groupe socioprofessionnel qui, en position d'intermédiaire informationnel, entretient des liens avec les professionnels de l'information et de la communication. En tant qu'insiders, ils disposent déjà de carnets d'adresses fournis qui peuvent se révéler très utiles en pareille occasion.

Ainsi, sur les conseils des plateformes, plusieurs interviewés expliquent avoir multiplié les contacts, virtuels et physiques, auprès des rédactions (parisiennes) dans l'intention de faire connaître leur cause. C'est le cas des salariés de Nice Matin, dont l'un d'entre eux s'est rendu à Paris pour faire le tour des rédactions la veille du lancement de la campagne de financement et de communication. En outre, les médias, qui se diversifient, se relancent ou tentent de survivre, possèdent déjà des «fichiers clients » que certains estiment comporter jusqu'à 100000 entrées. Du côté de Society, la rédaction dispose de la page Facebook de SoFoot (publication sœur) qui affiche plus de 600000 suiveurs au moment de la campagne (début 2015).

Cette capacité à mobiliser du capital symbolique afin d'obtenir des ressources économiques peut également être un gage de crédibilité pour convaincre des banquiers ou des mécènes de participer (à leur tour) à un projet (journalistique). En d'autres termes, un investisseur institutionnel ou privé évaluera d'autant mieux le niveau de risque après que des centaines ou des milliers de gens ont consenti à donner de l'argent pour un (futur) média. L' " économie de la confiance » (Laurent, 2012 ; Luhmann, 2006) joue alors à plein, ce qui permet aux médias les plus expérimentés d'accroître leur capacité à boucler leur montage financier. Ce faisant, cela vient créditer la thèse des avantages cumulés (" effet Matthieu »). En effet, de multiples aspects du financement participatif démontrent les divers avantages dont jouissent les agents ou les structures dominants pour engranger des recettes par le financement participatif (Nice Matin, Society, Hors-Série, Terra $E c o)$. Ces derniers, déjà établis, disposent d'un capital 


\section{Guillaume GOASDOUÉ}

symbolique non négligeable ainsi que de moyens pour professionnaliser leur campagne: détacher des salariés, réaliser des vidéos, organiser des événements, etc. Autant de tâches que l'ensemble des interviewés a expliqué avoir sousestimées en amont des campagnes. En effet, à rebours d'une autre idée reçue, un moyen d'obtenir de l'" argent facile » (Hui, Gerber et Greenberg, 2012), les campagnes supposent un investissement constant que les petites structures peuvent difficilement assurer sur la durée. Une journaliste d'une équipe de six personnes, qui y a consacré tout son temps pendant les 40 jours de collecte, a voulu reprendre à son compte la formule qu'un responsable d'une plateforme lui avait soufflée : " c'est à la fois un sprint et un marathon ».

On comprend, dès lors, que la possession d'un capital multiforme facilite d'autant mieux le succès et l'ampleur des campagnes des titres et des journalistes qui ont déjà fait leurs preuves par le passé et ont constitué, ne serait-ce qu'à l'état virtuel, une communauté. Deux extraits d'entretien illustrent ce principe :

« je pense qu'on a capitalisé sur une notoriété et une légitimité qu'on avait acquises [...] parce qu'on avait travaillé dix ans à Arrêt-sur-images. Donc évidemment, ça a permis de faire connaître notre travail, notre positionnement, et c'est essentiellement ça qui a permis de [nous] lancer » (Horssérie).

" la comm[unication] est plutôt un truc qu'on maîtrise et sur lequel on a quelques atouts, et on a du réseau, vraiment, c'est un de nos... c'est notre capital... capital de départ » (Les jours).

\section{Conclusion}

Cet article analyse l'émergence du financement participatif pour le cas du journalisme en France. Après avoir introduit le sujet en précisant quelques spécificités et enjeux sociologiques, économiques et politiques, nous avons discuté de la littérature internationale, encore partielle sur ce sujet, puis avons évoqué une quinzaine de cas. Deux aspects ont ensuite été abordés afin de mieux comprendre la nature et les mécanismes du financement participatif. Le premier éclairage a mis en lumière le rôle décisif du capital accumulé, lequel est mobilisé pour 
Analyse sociologique et économique du financement participatif. Ressorts et critiques dans le cas du journalisme

(2010-2015)

parvenir à lever des fonds. Le deuxième éclairage a porté sur la dimension sociale et communicationnelle du procédé. Chemin faisant, les résultats ont permis de nuancer quelques idées reçues (panacée économique, foule de contributeurs, easy money).

En définitive, le système rend compte du pouvoir de consécration des médias établis qui, d'une part, participent grandement à la communication autour des campagnes de financement participatif et, d'autre part, sont à l'origine de la notoriété des journalistes qui lancent avec le plus de succès de nouveaux médias sur ces plateformes. Le système tend donc à favoriser les agents déjà dominants du champ de l'information, ceux que leur habitus professionnel prédispose le plus à jouer le jeu du financement participatif (tel qu'il est décrit ci-dessus), alors même que le procédé n'est pas exempt de critiques (intermédiaires aux activités lucratives, surplus de travail, idéologie marchande et mercatique, dépolitisation, etc.).

En guise de prolongement, il serait intéressant de tirer un premier bilan, après plusieurs années, afin de voir en quoi cette alternative contribue (ou non) à apporter une solution aux problèmes structurels du financement de l'information (Franklin, 2014). On pense notamment à l'échec du modèle économique des pure-players "gratuits " financés principalement par la publicité, mais aussi à la rigidité du système des aides publiques à la presse, lequel est fait de dispositions complexes et inadaptés, notamment pour les nouveaux entrants. Enfin, de manière plus générale, on peut $y$ voir une invitation à (re)discuter du rôle des instances gouvernementales dans le financement des secteurs médiatiques et culturels, secteurs marchands pas comme les autres.

\section{RÉFÉRENCES BIBLIOGRAPHIQUES}

AITAMURTO T., 2011, «The Impact of Crowdfunding on Journalism, Case study of Spot.Us, A Platform for Community-funded Reporting », Journalism Practice, vol. 5, $\mathrm{n}^{\circ} 4$, pp. 429-445.

ARTWICK C., 2013, « Reporters on Twitter », Digital Journalism, vol. $1, \mathrm{n}^{\circ} 2$, pp. $212-228$. 


\section{Guillaume GOASDOUÉ}

BENNET L., B. CHIN et B. JONES (dir.), 2015, "Crowdfunding », New Media \& Society, vol. 17, $\mathrm{n}^{\circ}$ 2, pp. 141-307.

BOCZKOWSKI P. et L. PEER, 2011, «The Choice Gap: The Divergent Online News Preferences of Journalists and Consumers ", Journal of Communication, vol. $61, \mathrm{n}^{\circ} 5$, pp. 857-876.

BOUQUILLION P. et J. T. MATTHEWS, 2010, Le Web collaboratif. Mutations des industries culturelles, Grenoble, Presses universitaires de Grenoble.

BOURDIEU P., 1980, "Le capital social », Actes de la recherche en sciences sociales, vol. 31, pp. 2-3.

BOURDIEU P., 1997, Méditations pascaliennes, Paris, Seuil.

BOURDIEU P., 2015, Sociologie générale (volume 1). Cours au Collège de France 1981-1983, Paris, Raisons d'agir/Seuil.

BRABHAM D., 2008, « Crowdsourcing as a Model for Problem Solving. An Introduction and Cases », Convergence: The International Journal of Research into New Media Technologies, vol. 14, $\mathrm{n}^{\circ}$ 1, pp. 75-90.

BRUNS A., 2012, " Journalists and Twitter: How Australian News Organisations Adapt to a New Medium », Media International Australia, $\mathrm{n}^{\circ} 142$, pp. 97-107.

BURTCH G., A. GHOSE A. et S. WATTAL, 2013, «An Empirical Examination of The Antecedents and Consequences of Investment Patterns in Crowd-funded Markets ", Information Systems Research, vol. 24, n³, pp. 499-519.

CAGÉ J., 2015, Sauver les médias. Capitalisme, crowdfunding et démocratie, Paris, Seuil.

CARVAJAL M., J. GARCIA-AVILES et J. GONZALEZ, 2012, "Crowdfunding and Non-Profit Media ", Journalism Practice, vol. 6, $\mathrm{n}^{\circ}$ 5-6, pp. 638-647.

CARDON D. et A. CASILLI, 2015, Qu'est-ce que le digital labor?, Paris, Éditions de l'INA.

DUJARIER M.-A., 2008, Le travail du consommateur. De McDo à eBay: comment nous coproduisons ce que nous achetons, Paris, La Découverte.

FRANKLIN B., 2014, "The Future Of Journalism. In An Age of Digital Media and Economic Uncertainty ॥, Digital Journalism, vol. 2, $\mathrm{n}^{\circ} 3$, pp. 254-272.

GERBER E., J. HUI et P.-Y. KUO, 2012, « Crowdfunding: Why People Are Motivated to Post and Fund Projects on 
Analyse sociologique et économique du financement participatif. Ressorts et critiques dans le cas du journalisme

(2010-2015)

Crowdfunding Platforms ", Conference Computer Supported Cooperative Work 2012.

GERLITZ C. et A. HELMOND, 2013, « The Like Economy: Social Buttons and the Data-intensive Web », New Media \& Society, vol. 15, $\mathrm{n}^{\circ}$ 8, pp. 1348-1365.

GOASDOUÉ G., 2016, « Le recours au financement participatif par les médias d'information: levier de communication, travail en soi, idéologie marchande », Questions de communication, $\mathrm{n}^{\circ}$ 29, Varia, pp. 289-306.

GOYANES M., 2014, "An Empirical Study of Factors that Influence the Willingness to Pay for Online News ", Journalism Practice, vol. 8, $\mathrm{n}^{\circ}$ 6, pp. 742-757.

GUIDICI G., M. GUERINI et C. ROSSI-LAMASTRA, 2013, « Why Crowdfunding Projects can Succeed: The Role of Proponents' Individual and Territorial Social Capital », April 24, <http://ssrn.com/abstract=2255944>, dernière consultation le 24 février 2017.

HESMONDHALGH D., 2010, « User-generated Content, Free Labour and the Cultural Industries ", Ephemera: Theory and Politics in Organization, vol. 10, $n^{\circ} 3-4$, pp. 267-284.

HUI J., E. GERBER et M. GREENBERG, 2012, « Easy Money? Demands of Crowdfunding Work », Segal Technical Report, $\mathrm{n}^{\circ} 4$, <https://pdfs.semanticscholar.org/e81b/020c5ec52229f9c12 cab8c40c986222d2ff7.pdf> dernière consultation le 24 février 2017.

HUNTER A., 2015, « Crowdfunding Independant and Freelance Journalism: Negociating Journalistic Norms of Autonomy and Objectivity ", New Media \& Society, vol. 17, $\mathrm{n}^{\circ} 2$, pp. 272-288.

JIAN L. et J. SHIN, 2014, "Motivations Behind Donors' Contributions to Crowdfunded Journalism », Mass Communication and Society, $\mathrm{n}^{\circ} 18, \mathrm{pp} .165-185$.

JIAN L. et N. USHER, 2014, "Crowd-Funded Journalism », Journal of Computer-Mediated, vol. 19, $\mathrm{n}^{\circ} 2$, pp. 155-170.

LASORSA D., S. LEWIS et A. HOLTON, 2012, " Normalizing Twitter: Journalism Practice in an Emerging Communication Space ", Journalism Studies, vol. 13, n 1, pp. 19-36.

LAURENT E., 2012, Économie de la confiance, Paris, La Découverte "Repères ".

LUHMANN N., 2006 [1968], La confiance, un mécanisme de réduction de la complexité sociale, Paris, Economica. 


\section{Guillaume GOASDOUÉ}

MATTHEWS J. T., V. ROUZÉ et J. VACHET, 2014, La culture par les foules? Le crowdfunding et le crowdsourcing en question, Paris, MKF éditions.

MAUSS M., 2007 [1925], Essai sur le don. Forme et raison de l'échange dans les sociétés archaïques, Paris, Presses universitaires de France.

MERCKLÉ P., 2016, Sociologie des réseaux sociaux, Paris, La Découverte.

REBILLARD F. et N. SMYRNAIOS, 2010, "L'actualité selon Google. L'emprise du principal moteur de recherche sur l'information en ligne ", Communication et langages, $\mathrm{n}^{\circ} 160$, pp. 95-108.

SANCHEZ-GONZALEZ M. et M. PALOMO-TORRES, 2014, «Knowledge and Assessment of Crowdfunding in Communication. The View of Journalists and Future Journalists », Comunicar, n 43, pp. 101-110.

SCHOLZ T. (dir.), 2012, Digital Labor: The Internet as Playground and Factory, New York, Routledge.

SINGER J., 2014, "User-Generated Visibility: Secondary Gatekeeping in a Shared Media Space », New Media and Society, vol. 16, $\mathrm{n}^{\circ} 1$, pp. 55-73.

VEYNE P., 1976, Le pain et le cirque. Sociologie historique d'un pluralisme politique, Paris, Seuil.

VIS F., 2013, "Twitter As a Reporting Tool for Breaking News », Digital Journalism, vol. 1, $\mathrm{n}^{\circ} 1$, pp. 27-47. 
Analyse s`ci’ I’ gique et éc` $n `$ mique du financement participatif. Ress` rts et critiques dans le cas du ju urnalisme (2010-2015)

\section{Annexe 1 - Liste des pr jets}

\begin{tabular}{|c|c|c|c|c|c|c|}
\hline $\begin{array}{l}\mathrm{N} ` \mathrm{~m} \text { du } \\
\text { média }\end{array}$ & Descripti` $n$ & $\begin{array}{l}\text { Date de fin } \\
\text { de c'llecte }\end{array}$ & $\begin{array}{l}\text { M`ntant } \\
\text { en eur`s }\end{array}$ & $\mathrm{P}^{\prime}$ urcentage & $\begin{array}{l}\text { N`mbre de } \\
c^{`} \text { ntributi ns }\end{array}$ & Adresse URL \\
\hline Aqui & $\begin{array}{l}\text { Site régi` nal « gratuit » } \\
\text { év` luant vers le } \\
\text { (semi)payant en } 2015\end{array}$ & $31 / 01 / 2015$ & 31885 & 35 & 209 & $\begin{array}{l}\text { https://aqui.mipise.c’ m/fr/url_url-pr` ject- } \\
\underline{215441485}\end{array}$ \\
\hline Brief.me & $\begin{array}{l}\text { Lettre d'inf' rmati` } n \\
\text { qu' tidienne payante } \\
\text { (digest) }\end{array}$ & $10 / 11 / 2014$ & $\begin{array}{l}\text { envir`n } \\
20000\end{array}$ & 177 & 886 & http://fr.ulule.c' m/briefme/ \\
\hline $\begin{array}{l}\text { C'urrier } \\
\text { des } \\
\text { Balkans }\end{array}$ & $\begin{array}{l}\text { Site d'inf' rmati` n év' luant } \\
\text { vers le m’ dèle payant } \\
\text { (2015) }\end{array}$ & $06 / 11 / 2014$ & 21357 & 106 & 448 & http://fr.ulule.c' m/cdbalkans/ \\
\hline $\begin{array}{l}\text { Enquête } \\
\text { uverte (1) }\end{array}$ & $\begin{array}{l}\text { Site d'investigati` } n \\
\text { Enquêtes financées au } \\
c^{\prime} \text { up par c' up }\end{array}$ & $23 / 06 / 2013$ & 7700 & 140 & 146 & $\begin{array}{l}\text { http://www.kisskissbankbank.c' m/residences-de- } \\
\text { t' urisme-placement-t` xique }\end{array}$ \\
\hline $\begin{array}{l}\text { Enquête } \\
\text { uverte (2) }\end{array}$ & $\begin{array}{l}\text { Site d'investigati` } n \\
\text { Enquêtes financées au } \\
c^{\prime} \text { up par c' up }\end{array}$ & $29 / 06 / 2014$ & 8060 & 146 & 183 & http://fr.ulule.c' m/decharges/ \\
\hline Hexag`nes & $\begin{array}{l}\text { Site d'inf rmati` } n \\
\text { généraliste payant }\end{array}$ & $20 / 06 / 2014$ & 17265 & 115 & 333 & $\begin{array}{l}\text { http://www.kisskissbankbank.c` m/hexag` nes-l- } \\
\text { aventure-du-n` uveau-j’ urnalisme }\end{array}$ \\
\hline $\mathrm{H}$ rs-série & $\begin{array}{l}\text { Site payant, entretiens } \\
\text { vidé }\end{array}$ & $28 / 04 / 2014$ & 76416 & 127 & 3188 & http://fr.ulule.c` m/h`rs-serie/ \\
\hline $\begin{array}{l}\text { Kaïzen } \\
\text { (sais`n 2) }\end{array}$ & $\begin{array}{l}\text { Magazine bimestriel payant } \\
\text { et site web }\end{array}$ & $08 / 03 / 2015$ & 70838 & 118 & 1797 & http://www.kisskissbankbank.c` m/kaizen-sais`n-2 \\
\hline $\begin{array}{l}\text { La France } \\
\text { vue d'ici } \\
\text { (1) }\end{array}$ & $\begin{array}{l}\text { Site " gratuit » de } \\
\text { ph`t" rep` rtages }\end{array}$ & $28 / 07 / 2014$ & 24006 & 120 & 546 & $\begin{array}{l}\text { http://www.kisskissbankbank.c' m/la-france-vue-d- } \\
\text { ici }\end{array}$ \\
\hline $\begin{array}{l}\text { La France } \\
\text { vue d'ici } \\
\text { (2) }\end{array}$ & $\begin{array}{l}\text { Site " gratuit » de } \\
\text { ph`t rep` rtages }\end{array}$ & $09 / 06 / 2015$ & 16061 & 107 & 285 & $\begin{array}{l}\text { www.kisskissbankbank.c' m/la-france-vue-d-ici- } \\
\text { acte-ii }\end{array}$ \\
\hline
\end{tabular}




\begin{tabular}{|c|c|c|c|c|c|c|}
\hline Les Jours & $\begin{array}{l}\text { Site payant (lancement } \\
\text { prévu fin 2015) }\end{array}$ & $13 / 07 / 2015$ & 80175 & 160 & 1456 & $\begin{array}{l}\text { http://www.kisskissbankbank.com/les-jours-se- } \\
\text { levent-avec-vous }\end{array}$ \\
\hline Mars Actu & $\begin{array}{l}\text { Site régional « gratuit » } \\
\text { évoluant vers le } \\
\text { (semi)payant en } 2015\end{array}$ & $10 / 07 / 2015$ & 44340 & 177 & 839 & http://fr.ulule.com/marsactu/ \\
\hline Nice Matin & $\begin{array}{l}\text { Quotidien régional payant } \\
\text { et site web }\end{array}$ & $20 / 09 / 2014$ & 376275 & 125 & 2733 & http://fr.ulule.com/sauvons-nicematin/ \\
\hline $\begin{array}{l}\text { Paroles de } \\
\text { conflits }\end{array}$ & $\begin{array}{l}\text { Documentaire et } \\
\text { webdocumentaire }\end{array}$ & $31 / 10 / 2010$ & 18550 & 103 & 181 & $\begin{array}{l}\text { http://www.kisskissbankbank.com/paroles-de- } \\
\text { conflits }\end{array}$ \\
\hline $\begin{array}{l}\text { Rue89- } \\
\text { Strasbourg }\end{array}$ & $\begin{array}{l}\text { Site régional " gratuit » } \\
\text { évoluant vers le } \\
\text { (semi)payant en } 2015\end{array}$ & $26 / 06 / 2015$ & 36395 & 103 & 867 & http://fr.ulule.com/rue89-strasbourg/ \\
\hline Society & $\begin{array}{l}\text { Quinzomadaire papier } \\
\text { payant et site web }\end{array}$ & $01 / 03 / 2015$ & 50199 & 795 & 936 & $\begin{array}{l}\text { http://www.kisskissbankbank.com/society-abonne- } \\
\text { fondateur }\end{array}$ \\
\hline Terra Eco & Mensuel payant et site web & $10 / 10 / 2014$ & 105106 & 105 & 1668 & http://fr.ulule.com/terraeco/ \\
\hline
\end{tabular}

